\title{
Matrix elements and baryon spectroscopy from unquenched lattice QCD with improved staggered quarks.
}

E. B. Gregory, A. Irving, C. McNeile*, S. Miller, and Z. Sroczynski.

Department of Mathematical Sciences,

University of Liverpool,

Liverpool

L69 3BX, UK

E-mail: meneiledamtp.liv.ac.uk

We look for the effect of open decay channels on the masses of baryons in unquenched lattice QCD. We apply variational smearing using fuzzed basis states to a staggered nucleon operator. The signal for $\bar{s} s$ current in the nucleon is studied. The lattice calculations are done using improved staggered fermions.

XXIIIrd International Symposium on Lattice Field Theory

25-30 July 2005

Trinity College, Dublin, Ireland

${ }^{*}$ Speaker. 


\begin{tabular}{|c|c|c|}
\hline Baryon & Numbers & decay channel \\
\hline $\mathrm{N}(1535)$ & $S_{11}$ & $N \pi, N \eta$ \\
$\mathrm{N}(1440)$ & $P_{11}$ & $N \pi, \ldots$ \\
$\Lambda(1405)$ & $S_{01}$ & $\Sigma \pi, \ldots$ \\
$\Delta(1232)$ & $P_{33}$ & $N \pi$ \\
\hline
\end{tabular}

\section{Introduction}

There are a number of issues and questions about baryons that can be addressed using lattice QCD. For example the level ordering between the mass of the parity partner of the nucleon and the mass of the first excited state of the nucleon. Also as we discuss in section 3 there are matrix elements of the nucleon, such as the scalar current of strange quarks in the nucleon that have an uncertainty of at least a factor of 3. In this paper we make a start to study the mass of the parity partner of the nucleon and develop lattice techniques to compute the strangeness content of the nucleon using staggered fermions.

Seduced by the light sea quark masses and large volumes of the unquenched lattice QCD calculations performed by the MILC collaboration [1, 2], we use the improved staggered fermion formulation (known as "Asqtad") to do our calculations. The unquenched calculations use $2+1$ flavours of sea quarks with quark masses of $0.007 / 0.05$. The lattice volume was $20^{3} 64$ and $\beta=$ 6.76. We used the gauge configurations generated by the MILC collaboration stored on the public NERSC archive.

\section{The effect of open decays on the lattice.}

Many of the really interesting questions in hadronic physics, such as the existence of hybrid mesons or glueballs, requires lattice QCD to be able to cope with hadrons that decay via the strong interaction. Recent unquenched lattice QCD calculations now have light enough sea quarks that hadrons are starting to decay. The problem is most severe for particles that decay via S-wave decays, because particles that decay via $\mathrm{P}$-wave channels have additional kinematic obstacles because the decay products have to have momentum and this can be quite large on typical lattices [3].

Indeed the MILC collaboration have claimed to see problems for the $a_{0}$ state [1], 2] and $1^{-+}$ hybrid meson [П], due to the quark masses being light enough for these particles to decay into two mesons. There have also been problems reported with the singlet $0^{++}$mesons [5]. The experimental status of the above mesons is confusing, so it would be better to study the effect of hadronic decays on hadrons where there is less uncertainty about the experimental data, So it is important to study the issue of open decay channels of baryons.

The masses of baryons quoted in the particle data table (PDG) [6] are usually extracted using a partial waves analysis, hence the angular momentum of the decay can be read off from the quantum numbers. In the table 2 we summarise some data about some baryons from the PDG [6]. From table 2 the decay of the parity partner of the nucleon (N(1535)) looks like a good candidate to have the open decay of $N \pi$, in the current generation of unquenched calculations.

There has been a lot of work on using quenched QCD to study excited baryons. It has been found that large volumes $(>3 \mathrm{fm})$ are required, as well as light quark masses for the chiral extrap- 
olation [7]. At the moment only the improved staggered program of the MILC collaboration comes close to these requirements. Although in this calculation we have used a lattice with a physical box side of $2.4 \mathrm{fm}$, the MILC collaboration [2] have also generated unquenched gauge configurations with a box size of $3.4 \mathrm{fm}$.

Unfortunately, the identification of baryon operators with physical states is non-trivial in the staggered formalism [8]. The staggered baryon operators that are local in time break down into irreducible representations like: $5.8+2.8^{\prime}+4.16$ [8]. The nucleon couples to 8 and 16 representations [8]. Things are more complicated for the parity partner states. The $\Lambda(1405) \frac{1}{2}^{-}$will couple to the odd 8 representation. There are no interpolating operators for staggered fermions that couple to the parity partner of the nucleon in the ground state.

The staggered parity partner to the nucleon with mass $m_{-}$is extracted from the correlator $c(t)$ using the fit model in equation 2.1 .

$$
c(t)=a_{+} \exp \left(-m_{+} t\right)+(-1)^{t} a_{-} \exp \left(-m_{-} t\right)
$$

where $m_{+}$is the mass of the nucleon. There have been earlier attempts to extract the physical $m_{-}$ with unimproved staggered fermions [9].

In figure 1 we plot the masses $m_{+}$and $m_{-}$in lattice units as a function of the starting time in the fit. We used the gauge configurations from the MILC collaboration mentioned in the introduction and used wall sources in Coulomb gauge to generate the quark propagators. The actual number of configurations is in table 1. We also include the published number for $m_{N+}$ mass from MILC (they don't publish numbers for $m_{N_{-}}$) as well as the sum of the nucleon mass and the mass of the Goldstone pion.

The data in the figure 1 are consistent with $m_{N-}=m_{N+}+m_{\pi}$. However, the box side has a physical length of $2.4 \mathrm{fm}$, so the $m_{N-}$ mass may be suppressed by finite volume effects. In physical units the mass of the Goldstone pion is $310 \mathrm{MeV}$ in this calculation. The physical mass splitting between the nucleon and $\mathrm{N}(1535)$ state is about $600 \mathrm{MeV}$, so the state with the opposite parity in the nucleon channel is allowed energetically (at least) to decay to a nucleon and pion. A careful study of the $m_{N-}$ mass in larger volumes and with heavier quark masses (where the decay is closed) is required to confirm that this state has decayed.

We are not very comfortable with using equation 2.1 to extract physical numbers for $m_{N-}$, because multiple exponential fits to a single channel can be unstable. Also to study the issue of particle decay we need as many excited states as possible. So we have started to investigate using a basis of interpolating operators $\left(\Phi_{i}(t)\right)$ and extracting the masses and amplitudes via a variational technique.

A matrix of correlators is measured

$$
c(t)_{i j}=\left\langle\Phi_{i}(t) \Phi_{j}(0)^{\dagger}\right\rangle
$$

and analysed with a fit model of the form

$$
c(t)=A^{\dagger} T A
$$

For staggered fermions, the diagonal matrix $T$ contains exponentials $\left(e^{-m_{+} t}\right)$ and exponentials with prefactors of $(-1)^{t}$. We prefer to fit the data to the expression in equation 2.3, rather than use techniques such as the generalised eigenvalue method. 
Mass of nucleon and opposite parity state

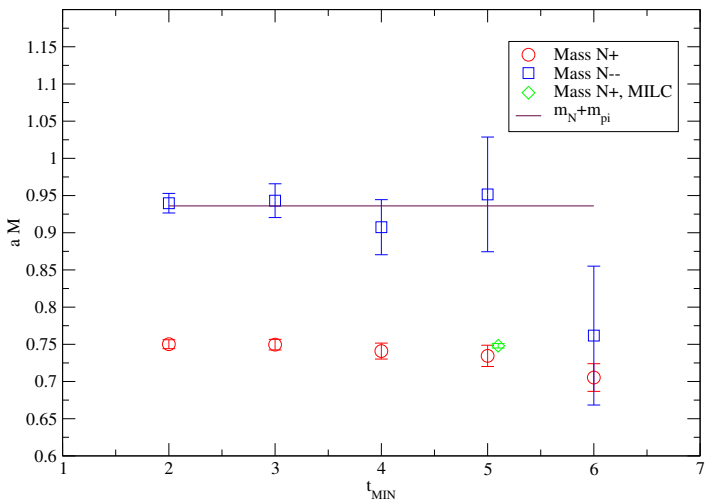

Figure 1: Mass of the nucleon and the opposite parity state that couples to the same correlator as a function of the first timeslice in the fit.2.1

\begin{tabular}{|c|c|c|c|c|}
\hline Smearing & No. & Region & a $m_{+}$ & a $m_{-}$ \\
\hline Wall & $2 \times 342$ & $4-15$ & $0.74(1)$ & $0.91(4)$ \\
Variational & $1 \times 230$ & $4-15$ & $0.84(2)$ & $1.1(5)$ \\
Wall, MILC & $8 \times 493$ & $5-14$ & $0.7480(30)$ & \\
\hline
\end{tabular}

Table 1: Fit results for the nucleon and the state with opposite parity in the same channel. The number before the $\times$ symbol is the number of time sources for the quark propagator calculations on each configuration

We used "fuzzed" and local basis states [10] originally developed for clover fermions. To respect the staggered symmetries fuzzed gauge links only separate the quark and anti-quark by multiples of 2 . Here we try it for the local staggered nucleon operator that belongs to the 8 representation.

$$
O(t)=\sum_{x \text { even }} \varepsilon^{a b c} \chi_{a}(x, t) \chi_{b}(x, t) \chi_{c}(x, t)
$$

The Chroma software system was used for the lattice calculations [11].

In table 1 we show some results for the variational analysis versus using wall sources from MILC's analysis and our analysis. The variational analysis is inconsistent at the $4 \sigma$ level with the results that use single wall sources. For Wilson fermions, wall sources have small statistical errors, but tend to approach a plateau very slowly. For this data set MILC fitted the nucleon operator at smaller times than for the equivalent analysis at other $\beta$ values. It is not clear what causes the difference between the mass from the wall source and variational analysis.

\section{Strangeness content the nucleon}

The matrix element $\langle N|\bar{s} s| N\rangle$ is colloquially known as the strangeness content of the nucleon. This matrix element is important for baryon chiral perturbation theory. This matrix element is also used in the neutralino-nucleon scalar cross-section [12] and hence crucial for the direct detection 


\begin{tabular}{|c|c||c|c|c|}
\hline Group & $y$ & Group & $n_{f}$ & $y$ \\
\hline Borasoy and Meißner [14] & $0.21 \pm 0.20$ & Kentucky [15] & 0 & $0.36(3)$ \\
Borasoy [16] & $0.20 \pm 0.12$ & SESAM [17] & 2 & $0.59(13)$ \\
John Ellis [18] & $\sim 0.6$ & UKQCD [19] & 2 & $-0.28(33)$ \\
\hline
\end{tabular}

Table 2: Some lattice and non-lattice determinations of $y$.

of dark matter. It is convenient to discuss the ratios of matrix elements

$$
y=2 \frac{\langle N|\bar{s} s| N\rangle}{\langle N|\bar{u} u+\bar{d} d| N\rangle}
$$

where $N$ is the nucleon operator.

In the table 2 we record some recent values for $y$ from lattice and non-lattice methods. There has also been a large quenched study of matrix elements related to $y$ by Lewis et al. [13]. The value of $y$ can range from 0.2 to 0.6 . The $\langle N|\bar{s} s| N\rangle$ matrix element should not be effected by open decay channels, so a precision value should be attainable.

On the lattice the matrix element $\langle N|\bar{s} s| N\rangle$ is extracted from the correlation of a strange loop with the two point nucleon correlator [17, 19]. The loops were computed via a standard stochastic technique using random noise sources [20]. With staggered fermions the scalar loop has no phase factors [5]. However, the nucleon two point correlator does have a contribution that oscillates in time (see equation 2.1). We used the unquenched data set described in section 1 1 with 330 gauge configurations. Quark propagators inverted from wall sources were used.

The strange loop is inserted at time $t_{1}$. The nucleon interpolating operators are at times 0 and $t$ with $t=t_{1}+t_{2}$. The fit model for the three point correlator is equation 3.2 .

$$
C_{3}^{(a b)}\left(t_{1}, t_{2}\right)=\sum_{i, j} c_{i}^{(a)} e^{-M_{i} t_{1}} x_{i j} e^{-M_{j} t_{2}} c_{j}^{(b)}
$$

where $a$ and $b$ label the interpolating operators and the time oscillating term is suppressed. The amplitudes $c_{j}^{(b)}$ and masses $M_{i}$ are fixed from the fits to the two point function. The $\langle N|\bar{s} s| N\rangle$ matrix element is proportional to $x_{00}$. In these preliminary fits we assumed $x_{01}=0$, but varied $x_{00}$ and $x_{11}$. Using $t_{1}=4$ and fitting from $\mathrm{t}=9$, we get $x_{00}=1.5 \pm 1.0$. For this data set, the two point nucleon correlator gets very noisy for $t>12$. Given the large statistical errors we do not quote a physical number for $\langle N|\bar{s} s| N\rangle$ or $y$.

\section{Conclusions}

We have started to study baryons on the lattice when the decay channels are open. It is critical to include finite volume studies of these states [7]. This is computationally feasible with the current generation of improved staggered fermion calculations.

For poorly known quantities such as the strangeness content of the nucleon, we should be able to (finally) pin this matrix element down. The statistical error on the bare matrix element is disappointingly large, but we hope that by incorporating the variational analysis and using many more gauge configurations the errors should be reduced. 


\section{References}

[1] C. W. Bernard et. al., The qcd spectrum with three quark flavors, Phys. Rev. D64 (2001) 054506, [hep-lat/0104002].

[2] C. Aubin et. al., Light hadrons with improved staggered quarks: Approaching the continuum limit, Phys. Rev. D70 (2004) 094505, [hep-lat/ 0402030 ].

[3] C. Michael, Hadronic decays, PoS(LAT2005)008 (2005) hep-lat/0509023.

[4] C. Bernard et. al., Lattice calculation of 1-+ hybrid mesons with improved kogut-susskind fermions, Phys. Rev. D68 (2003) 074505, [hep-lat/0301024].

[5] E. Gregory, A. Irving, C.McNeile, S.Miller, and Z.Sroczynski, Scalar glueball and meson spectroscopy in unquenched lattice qcd with improved staggered quarks, PoS(LAT2005)02才(2005).

[6] Particle Data Group Collaboration, S. Eidelman et. al., Review of particle physics, Phys. Lett. $\mathbf{B 5 9 2}$ (2004) 1.

[7] K. Sasaki and S. Sasaki, Excited baryon spectroscopy from lattice qcd: Finite size effect and hyperfine mass splitting, Phys. Rev. D72 (2005) 034502, [hep-lat/0503026.

[8] M. F. L. Golterman and J. Smit, Lattice baryons with staggered fermions, Nucl. Phys. B255 (1985) 328.

[9] N. Ishizuka, M. Fukugita, H. Mino, M. Okawa, and A. Ukawa, Operator dependence of hadron masses for kogut-susskind quarks on the lattice, Nucl. Phys. B411 (1994) 875-902.

[10] UKQCD Collaboration, P. Lacock, A. McKerrell, C. Michael, I. M. Stopher, and P. W. Stephenson, Efficient hadronic operators in lattice gauge theory, Phys. Rev. D51 (1995) 6403-6410, [hep-lat/9412079].

[11] SciDAC Collaboration, R. G. Edwards and B. Joo, The chroma software system for lattice qcd, hep-lat/0409003.

[12] A. Bottino, F. Donato, N. Fornengo, and S. Scopel, Implications for relic neutralinos of the theoretical uncertainties in the neutralino nucleon cross-section, Astropart. Phys. 13 (2000) 215-225, [hep-ph/9909228].

[13] R. Lewis, W. Wilcox, and R. M. Woloshyn, The nucleon's strange electromagnetic and scalar matrix elements, Phys. Rev. D67 (2003) 013003, hep-ph/0210064.

[14] B. Borasoy and U.-G. Meissner, Chiral expansion of baryon masses and sigma-terms, Annals Phys. 254 (1997) 192-232, hep-ph/9607432].

[15] S. J. Dong, J. F. Lagae, and K. F. Liu, $\pi n \sigma$ term, $\bar{s}$ in nucleon, and scalar form factor - a lattice study, Phys. Rev. D54 (1996) 5496-5500, [hep-ph/9602259].

[16] B. Borasoy, Sigma-terms in heavy baryon chiral perturbation theory revisited, Eur. Phys. J. C8 (1999) 121-130, [hep-ph/9807453].

[17] TXL Collaboration, S. Gusken et. al., The pion nucleon sigma-term with dynamical wilson fermions, Phys. Rev. D59 (1999) 054504, [hep-lat/9809066].

[18] J. R. Ellis, Today's view on strangeness, Eur. Phys. J. A24S2 (2005) 3-10, [hep-ph/0411369].

[19] UKQCD Collaboration, C. Michael, C. McNeile, and D. Hepburn, The strangeness content of the nucleon, Nucl. Phys. Proc. Suppl. 106 (2002) 293-295, hep-lat/ 0109028.

[20] E. B. Gregory, A. C. Irving, C. McNeile, S. Miller, and Z. Sroczynski, Pseudoscalar singlet physics with staggered fermions, PoS(LAT2005)083 (2005) hep-lat/0509193. 